\title{
Anesthetic complications due to extremely low ankle blood pressure caused by peripheral arterial disease
}

\author{
Jae Chan Choi ${ }^{1}$, Sang Hun Lee ${ }^{2}$, Soon Yul Kim ${ }^{1}$, Jong Hyuk Lee ${ }^{1}$, and Kyu-Yong Jang ${ }^{1}$ \\ Departments of ${ }^{1}$ Anesthesiology and Pain Medicine, ${ }^{2}$ Thoracic and Cardiovascular Surgery, Yonsei University Wonju College of \\ Medicine, Wonju, Korea
}

We report a case of anesthetic complications that resulted when anesthesia was monitored by measuring the ankle blood pressure. Extremely low ankle blood led to the administration of vasoactive drugs, the appearance of premature ventricular complexes (PVCs), light anesthesia, and recall of intraoperative pain.

An 89-year-old man (60 kg, $168 \mathrm{~cm})$ was scheduled for open reduction and internal fixation for fracture of the surgical neck of the right humerus. His past medical history included a diagnosis of hypertension 50 years ago and a 40 pack-year smoking history. An electrocardiogram (ECG) revealed premature atrial complexes and anterolateral ischemia, and basic blood chemistry revealed elevated creatinine $(1.53 \mathrm{mg} / \mathrm{dl}$; reference range: $<1.4 \mathrm{mg} / \mathrm{dl})$.

When the patient arrived in the operating room, his blood pressure was measured at the left ankle, using an automatic sphygmomanometer. His initial ankle blood pressure, before induction, was 115/98 $\mathrm{mmHg}$, and heart rate was 65 beats/min. Anesthesia and surgery were performed in a supine position. Following preoxygenation, anesthesia was induced with thiopental $200 \mathrm{mg}$ and remifentanil infusion $0.1 \mu \mathrm{g} / \mathrm{kg} / \mathrm{min}$. Rocuronium $50 \mathrm{mg}$ was administered to facilitate orotracheal intubation with a cuffed tube. Anesthesia was maintained with sevoflurane $1.5-2.0 \%$ in a $50 \%$ oxygen and remifentanil infusion, with positive pressure ventilation in a circle system. The administration of anesthetic agents was adjusted, according to the patient's blood pressure.

After tracheal intubation, his ankle blood pressure was 93/24 $\mathrm{mmHg}$ and heart rate was 99 beats/min. Because the diastolic pressure was low, ephedrine $8 \mathrm{mg}$ was administered twice. However, his ankle systolic blood pressure decreased further; blood pressure was $73 / 28 \mathrm{mmHg}$ and heart rate was 75 beats/ min. In order to increase the blood pressure, the sevoflurane concentration and remifentanil infusion rate were decreased. The patient received an intravenous bolus of phenylephrine 60 $\mu \mathrm{g}$, plus a continuous infusion of phenylephrine at $1.5 \mu \mathrm{g} / \mathrm{kg} /$ min. The phenylephrine infusion was titrated, according to the blood pressure of the patient. PVCs appeared and lidocaine 60 mg was intravenously administered. The left radial artery was cannulated and blood pressure was continuously monitored. At that moment, the blood pressure measured, at the left radial artery, was 199/80 $\mathrm{mmHg}$, while the left ankle blood pressure was $93 / 43 \mathrm{mmHg}$. The sevoflurane concentration and remifentanil infusion rate were increased and the phenylephrine infusion was stopped. The patient's radial blood pressure decreased and the PVCs disappeared.

Thereafter, the left ankle and brachial blood pressures were simultaneously measured during anesthesia. The left ankle blood pressure $(62 / 40 \mathrm{mmHg})$ was lower than the left brachial blood pressure $(139 / 53 \mathrm{mmHg})$ and the right ankle blood pressure (104/87 mmHg). The left brachial blood pressure was used to monitor anesthesia, and the target systolic blood pressure was $<140 \mathrm{mmHg}$. However, because the left ankle blood pressure was extremely low (e.g. 39/15 mmHg 15 minutes after skin incision), in order to maintain blood flow to the left lower extremity, the left brachial blood pressure was intermittently kept high by decreasing the anesthetic concentrations. These anesthetic methods might result in light

Corresponding author: Jae Chan Choi, M.D., Ph.D., Department of Anesthesiology and Pain Medicine, Yonsei University Wonju College of Medicine, 162, Ilsan-dong, Wonju 220-701, Korea. Tel: 82-33-762-8402, Fax: 82-33-742-8198, E-mail: jaechan@yonsei.ac.kr

(c) This is an open-access article distributed under the terms of the Creative Commons Attribution Non-Commercial License (http:// creativecommons.org/licenses/by-nc/3.0/), which permits unrestricted non-commercial use, distribution, and reproduction in any medium, provided the original work is properly cited. 
anesthesia and recall of intraoperative pain. The total anesthetic duration and surgery duration were 115 and 70 minutes, respectively.

When the patient was fully awake, he complained of intraoperative recall and pain experienced during anesthesia. Ten days after surgery, the arterial Doppler waveform was measured, using ImexLab 3000DX (portable Doppler, Nicolet Vascular [formerly Imex Medical Systems], Golden, CO, USA).
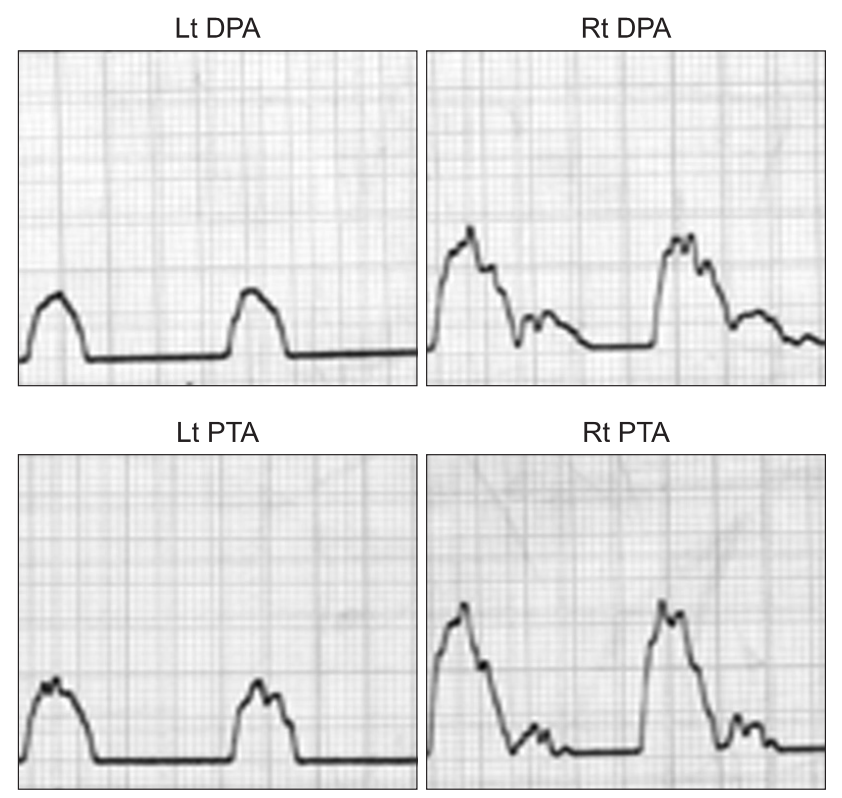

Lt PA
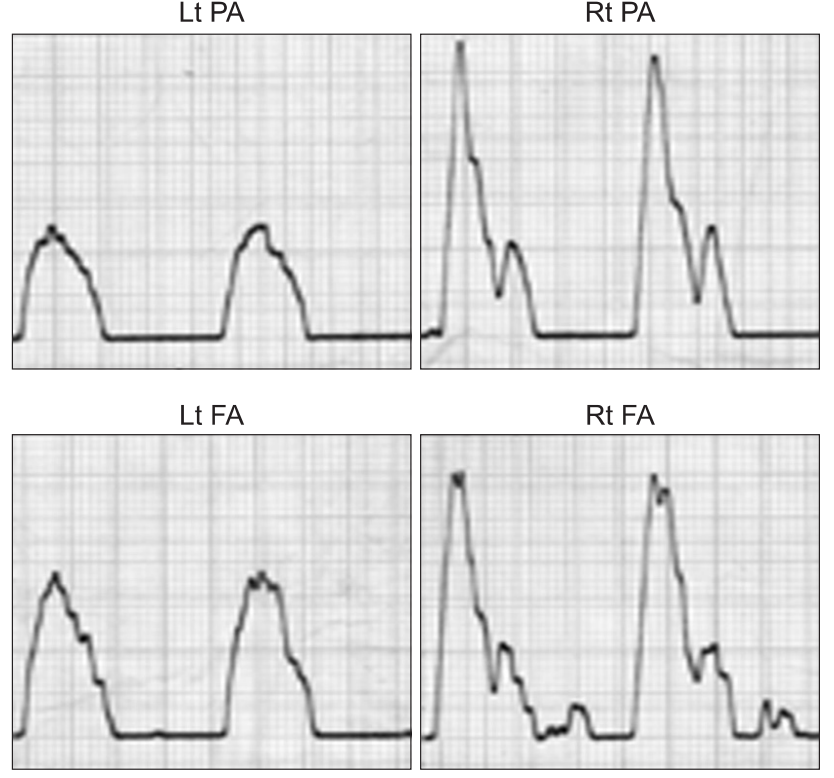

Fig. 1. Doppler waveforms measured bilaterally in four sites: the dorsalis pedis artery (DPA), posterior tibial artery (PTA), popliteal artery (PA), and femoral artery (FA). There are obstructed waveforms with diminished waveform height, rounding, and loss of diastolic flow reversal in the arteries on the left side, compared to those on the right side. Lt: left, Rt: right.
The Doppler waveform was measured bilaterally at four sites: the dorsalis pedis artery, posterior tibial artery, popliteal artery, and femoral artery. There were obstructed waveforms in the arteries on the left side, when compared to those on the right side with diminished waveform height, rounding of the waveform, and loss of diastolic flow reversal (Fig. 1). Because there were obstructed waveforms from the left dorsalis pedis artery to the left femoral artery, we suspect that the obstruction might exist between the aortic bifurcation of the left common iliac artery and the left femoral artery.

Peripheral arterial disease is chronic arterial occlusive disease of the lower extremities, which is caused by atherosclerosis [1]. The ABI (ankle-brachial index) is the ratio of the ankle to brachial systolic blood pressure. An ABI $<0.90$ indicates the presence of peripheral arterial disease [2]. An ABI between 0.90 and 0.71 indicates mild obstruction, between 0.70 and 0.41 moderate obstruction, and less than 0.41 indicate severe obstruction [3]. The ABI of the present case was 0.61 [77 (ankle SBP)/126 (brachial SBP)] at rest (ten days after surgery) and was 0.28 [39 (ankle SBP)/140 (brachial SBP)] ten minutes after skin incision in the operating room. Although not completely understood, the ABI decrease from 0.61 to 0.28 during anesthesia suggests that the obstruction, due to peripheral vascular disease, might have been aggravated during anesthesia. This also suggests that blood flow, distal to the obstruction site, should be adequately maintained during anesthesia when peripheral arterial disease exists.

A recent study has shown that inadequate blood pressure monitoring is associated with inadequate intraoperative blood transfusions, vasopressor infusions, and antihypertensive medication administration [4]. In the present case, inadequate blood pressure monitoring resulted in inadequate vasopressor infusion and the appearance of PVCs. PVCs may be caused by many medical conditions, including high blood pressure, stress, heart attack, and heart disease. Acute elevation in blood pressure may be associated with the generation of PVCs and the incidence of PVCs may be reduced by induced hypotension [5].

Ten days after the surgery, the left ankle blood pressure was $77 / 47 \mathrm{mmHg}$ and the left brachial blood pressure was 126/55 $\mathrm{mmHg}$. What was the reason that the left ankle blood pressure was 115/98 mmHg before induction? It may be that preoperative stress might increase the left ankle blood pressure before induction. If patient's brachial blood pressure was measured before induction, anesthesia might be delayed because of high brachial blood pressure.

In conclusion, this case suggests that brachial blood pressure should be measured before induction and during anesthesia in upper extremity operations, even if the intravenous line is in the unoperated upper extremity. During preoperative evaluation, anesthesiologists should be aware of the possibility 
of peripheral arterial disease in patients with risk factors, including old age, cigarette smoking, diabetes mellitus, and hypertension.

\section{References}

1. Aronow WS. Peripheral arterial disease in the elderly. Clin Interv Aging 2007; 2: 645-54.

2. Doobay AV, Anand SS. Sensitivity and specificity of the anklebrachial index to predict future cardiovascular outcomes: a systematic review. Arterioscler Thromb Vasc Biol 2005; 25: 1463-9.

3. Alonso A, McManus DD, Fisher DZ. Peripheral vascular disease. London, Jones and Bartlett publishers. 2011, pp 11-80.

4. Wax DB, Lin HM, Leibowitz AB. Invasive and concomitant noninvasive intraoperative blood pressure monitoring: observed differences in measurements and associated therapeutic interventions. Anesthesiology 2011; 115: 973-8.

5. Sideris DA, Kontoyannis DA, Michalis L, Adractas A, Moulopoulos SD. Acute changes in blood pressure as a cause of cardiac arrhythmias. Eur Heart J 1987; 8: 45-52. 